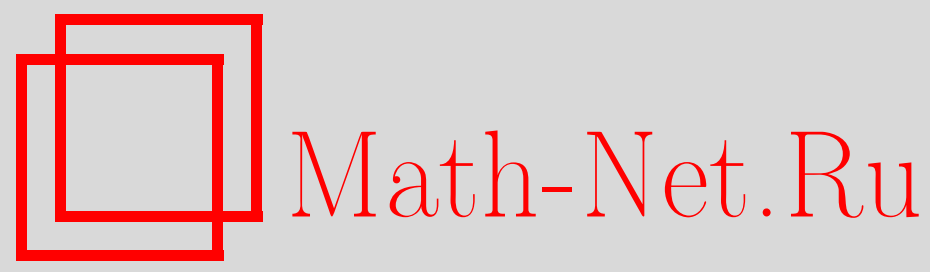

С. А. Егишянц, Е. И. Островский, Аппроксимация случайных полей обобщенными линейными сплайнами, Матем. заметки, 1998, том 63, выпуск 5, 690-696

DOI: https://doi.org/10.4213/mzm1335

Использование Общероссийского математического портала Math-Net.Ru подразумевает, что вы прочитали и согласны с пользовательским соглашением http://www . mathnet.ru/rus/agreement

Параметры загрузки:

IP : 3.85 .5 .30

26 апреля 2023 г., $16: 23: 07$

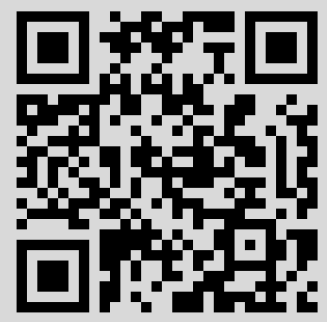




\title{
АППРОКСИМАЦИЯ СЛУЧАЙНЫХ ПОЛЕЙ ОБОБЩЕННЫМИ ЛИНЕЙНЫМИ СПЛАЙНАМИ
}

\author{
С. А. Егишянц, Е.И. Островский
}

В статье рассматривается задача о восстановлении случайного процесса или поля по известным его значениям в некоторой конечной сетке. Эта задача поставлена и решена в достаточно обшей форме, причем показано, что даже в простейшем случае аппроксимации случайного процесса обобщенньми линейными сплайнами нормированная должным образом погрешность приближения будет иметь экспоненциально убьвающий "хвост" распределения.

Библиография: 12 названий.

В данной статье рассматривается известная и часто встречающаяся на практике задача о восстановлении случайного процесса или поля (СП) по известным его значениям в некоторой конечной сетке. Ниже эта задача будет поставлена и решена в достаточно общей форме, причем будет показано, что даже в простейшем случае аппроксимации СП обобщенными линейными сплайнами нормированная должным образом погрешность приближения будет иметь экспоненциально убывающий “хвост" распределения.

В качестве возможных применений наших результатов упомянем задачи статистического анализа СП по значениям его реализаций в конечной сетке [1], а также метод Монте-Карло [2], [3].

1. Введение. Пусть $\xi(t)$ - сепарабельное центрированное случайное поле (СП), определенное, помимо вероятностного пространства, на некотором метрическом пространстве $(T, d), t \in T$. Предположим, что для поля $\xi(t)$ вьполняется условие Крамера равномерно по $t \in T$, т.е. существует константа $\lambda_{0}>0$ такая, что

$$
\sup _{\varepsilon= \pm 1, t \in T} \operatorname{E} \exp (\varepsilon \lambda \xi(t))<\infty
$$

при всех $\lambda \in\left(-\lambda_{0}, \lambda_{0}\right)$. Тогда на интервале $\lambda \in\left(-\lambda_{0}, \lambda_{0}\right)$ определена функция

$$
\varphi(\lambda)=\sup _{\varepsilon= \pm 1, t \in T} \ln E \exp (\varepsilon \lambda \xi(t)) .
$$

Легко видеть, что $\varphi(0)=0$ и, при этом, если $\lambda_{0}=\infty$, то $\varphi(\lambda) / \lambda \rightarrow \infty$ при $\lambda \rightarrow \infty$.

Согласно [4] и [5], с помощью функции $\varphi(\lambda)$ вводится банахово пространство случайных величин $\eta$ с конечной нормой

$$
\|\eta\|=\sup _{\varepsilon= \pm 1, \lambda \in\left(-\lambda_{0}, \lambda_{0}\right), \lambda \neq 0} \frac{\varphi^{-1}(\ln \mathrm{E} \exp (\varepsilon \lambda \eta))}{|\lambda|},
$$


где $\varphi^{-1}(x)$ - функция, обратная $\varphi(x)$.

Естественную метрику $d(t, s)$ на множестве $T$ определим выражением $d(t, s)=$ $\|\xi(t)-\xi(s)\|$. Преобразование Юнга-Фенхеля функции $\varphi(\lambda)[6]$ обозначим

$$
\varphi^{*}(x)=\sup _{\lambda \in\left(-\lambda_{0}, \lambda_{0}\right)}(\lambda x-\varphi(\lambda)) .
$$

Положим далее $\pi(u)=1 /\left(u \varphi^{* \prime}(u)\right)$.

Пусть $B(t, \delta)=\{s \in T: d(t, s) \leqslant \delta\}-d$-шар радиуса $\delta$ с центром в точке $t \in T$. Наименьшее число $d$-шаров радиуса $\varepsilon>0$, покрываюших некоторое подмножество $S$ пространства $T$, обозначим через $N(S, d, \varepsilon)$. Энтропией множества $S$ назьвается функция $H(S, d, \varepsilon)=\ln N(S, d, \varepsilon)$. Назовем поле $\xi(t)$ локально однородным на множестве $T$, если для любого $\varepsilon>0$

$$
h(\varepsilon)=\sup _{t \in T} \sup _{\delta>0} H(B(t, \delta), d, \delta \varepsilon)<\infty .
$$

Если, к примеру, $T$ - ограниченное вьпуклое подмножество $\mathbb{R}^{k}$ и $d(t, s) \sim d_{0}|t-s|^{\alpha}$ при $t-s \rightarrow 0, \alpha \in(0,1), d_{0}-$ константа, то $h_{0}^{-}+(k / \alpha) \ln (1 / \varepsilon) \leqslant h(\varepsilon) \leqslant h_{0}^{+}+(k / \alpha) \ln (1 / \varepsilon)$, и, следовательно, условие (1) выполнено.

Для решения поставленной задачи нужна оценка сверху распределения максимума случайного поля $\xi(t)$ при $t \in S \subset T$. Наиболее точные оценки приводятся в [7] и [8]:

Если $\xi(t)$ - локально однородное на множестве $(S, d) \mathrm{C \Pi},\|\xi(t)\|=1$ и сходится энтропийный ряд Дадли $\sum_{n} 2^{-n} h\left(S, d, 2^{-n}\right)$, то

где

$$
\mathrm{P}\left(\sup _{t \in S} \xi(t)>u\right) \leqslant C_{1} N(S, d, \pi(u)) e^{-\varphi^{*}(u)},
$$

$$
C_{1}=\exp \left(1+\inf _{0<p<1}(1-p) \sum_{n=1}^{\infty} p^{n} h\left(S, d, p^{n-1}\right)\right) .
$$

Пусть $S(\delta)$ - некоторая конечная сетка точек $t_{i}$ множества $T$,

$$
\delta=\sup _{t \in T} \min _{t_{i} \in S(\delta)} d\left(t, t_{i}\right)
$$

В $S(\delta)$ выделим подмножества $S_{t}(\delta)=\left\{t_{i} \in S(\delta): d\left(t, t_{i}\right) \leqslant C_{2} \delta\right\}, C_{2}>1$. Обозначим число элементов в $S_{t}(\delta)$ через $m(t, \delta)$. Константу $C_{2}$ разумно определять таким образом, чтобы вьполнялось соотношение $\inf _{t \in T} m(t, \delta)>1$. Если, к примеру, $T \subset \mathbb{R}^{k}$ и $d(t, s) \sim d_{0}|t-s|, t-s \rightarrow 0$, то целесообразно принять $C_{2}=2 \sqrt{k}$.

Обобщенным линейным сплайном для СП $\xi(t)$ назовем функцию

$$
\widehat{\xi}(t)=\sum_{i=1}^{m(t, \delta)} \alpha_{i}(t) \xi\left(t_{i}\right), \quad t_{i} \in S_{t}(\delta), \quad \alpha_{i}(t) \geqslant 0, \quad \forall t \in T \quad \sum_{i} \alpha_{i}(t)=1 .
$$

Мы не будем здесь конструктивно определять коэффициенты $\alpha_{i}(t)$ в $(3)$, желая подчеркнуть, что все полученные ниже результаты справедливы для любых сплайнов $\widehat{\xi}(t)$, представимых в виде (3). В качестве примера можно указать на регрессионные сплайны [9], а также классические сплайны [10, с. 131-132], для которых

$$
\alpha_{i}(t)=\frac{1}{m(t, \delta)-1} \sum_{t_{j} \in S_{t}(\delta), t_{j} \neq t_{i}} d\left(t, t_{j}\right) / \sum_{t_{j} \in S_{t}(\delta)} d\left(t, t_{j}\right)
$$

при $m(t)>1$. Обозначим еще

$$
\omega_{\xi}(\delta)=\sup _{t \in T}|\xi(t)-\widehat{\xi}(t)|
$$


2. Оценки погрешности сверху. Далее будут получены важные для практики оценки сверху распределения погрешности аппроксимации поля $\xi(t)$ сплайном $\widehat{\xi}(t)$ типа

$$
P_{T}(u)=\mathrm{P}\left(\sup _{\delta \in\left(0, \delta_{0}\right)} \frac{\omega_{\xi}(\delta)}{g(\delta)}>u\right) \leqslant C_{3} e^{-C_{4} u},
$$

где $g(\delta)$ - некоторая неслучайная функция, $\delta_{0}>0$, постоянные $C_{3}$ и $C_{4}$ не зависят от $u$. Параллельно выводится оценка верхнего предела вида

$$
\left.\varlimsup_{\delta \rightarrow 0} \frac{\omega_{\xi}(\delta)}{g(\delta)} \leqslant 1 \quad \text { (с вероятностью } 1\right)
$$

Докажем сначала две небольшие леммы.

ЛЕмМА 1. $\forall \gamma>1, \forall x$ выполнено $\varphi^{*}(\gamma x) \geqslant \gamma \varphi^{*}(x)$.

ДокАЗАТЕЛЬСТво. Пусть $\gamma>1$. Для вьпуклой функции $\varphi^{*}(x)$ по определению при любом $\alpha \in[0,1]$ и всех значениях $x_{1}$ и $x_{2}$ справедливо соотношение

$$
\varphi^{*}\left(\alpha x_{1}+(1-\alpha) x_{2}\right) \leqslant \alpha \varphi^{*}\left(x_{1}\right)+(1-\alpha) \varphi^{*}\left(x_{2}\right) .
$$

Выберем $x_{1}=0, x_{2}=\gamma x, \alpha=1-1 / \gamma$. Тогда, учитывая тот факт, что $\varphi^{*}(0)=0$, имеем $\varphi^{*}(x) \leqslant \varphi^{*}(\gamma x) / \gamma$, т.е. $\varphi^{*}(\gamma x) \geqslant \gamma \varphi^{*}(x)$. Таким образом, лемма 1 доказана.

Лемма 2. Справедливо неравенство

$$
\sup _{t \in T}\|\xi(t)-\widehat{\xi}(t)\| \leqslant C_{2} \delta
$$

ДокАЗАТЕЛЬСТво. Согласно формуле (3)

$$
\begin{aligned}
& \sup _{t \in T}\|\xi(t)-\widehat{\xi}(t)\|=\sup _{t \in T}\left\|\xi(t)-\sum_{i=1}^{m(t, \delta)} \alpha_{i}(t) \xi\left(t_{i}\right)\right\|=\sup _{t \in T}\left\|\sum_{i=1}^{m(t, \delta)} \alpha_{i}(t)\left(\xi(t)-\xi\left(t_{i}\right)\right)\right\| \\
& \quad \leqslant \sup _{t \in T}\left\|\max _{t_{i} \in S_{t}(\delta)}\left|\xi(t)-\xi\left(t_{i}\right)\right| \sum_{i=1}^{m(t, \delta)} \alpha_{i}(t)\right\| \leqslant \sup _{t \in T} \max _{t_{i} \in S_{t}(\delta)}\left\|\xi(t)-\xi\left(t_{i}\right)\right\| \leqslant C_{2} \delta
\end{aligned}
$$

что и требовалось доказать.

Обозначим $g(\delta)=C_{2} \delta \varphi^{*-1}\left(h\left(C_{2} \delta\right)\right)$, где $\varphi^{*-1}(x)$ - функция, обратная $\varphi^{*}(x)$.

TEOPEMA 1. Пусть

а) $C \Pi \xi(t)$ локально однородно на множестве $T$,

б) $\sum_{n} 2^{-n} h\left(2^{-n}\right)<\infty$,

в) $h(\pi(u))=o\left(\varphi^{*}(u)\right)$ nрu $u \rightarrow \infty$,

г) существует последовательность $y_{n} \downarrow 0$ при $n \rightarrow \infty$ такая, что

$$
\lim _{n \rightarrow \infty} \frac{y_{n}}{y_{n-1}}=1
$$

$u \forall \varepsilon>0 \quad \sum_{n} \exp \left(-\varepsilon h\left(y_{n}\right)\right)<\infty$.

Тогда справедливы неравенства (4) $и(5)$, где $C_{3}$ и $C_{4}$ определяются ниже. 
ДокАЗАТЕЛЬСТВо. Пусть $\delta_{n} \downarrow 0$ при $n \rightarrow \infty$. Обозначим

$$
P_{n}(u)=\mathrm{P}\left(\sup _{\delta \in\left(\delta_{n}, \delta_{n-1}\right)} \frac{\omega_{\xi}(\delta)}{g(\delta)}>u\right) .
$$

Оценим вероятность $P_{n}(u)$ сверху с помошью формулы $(2)$ :

$P_{n}(u) \leqslant \mathrm{P}\left(\frac{\omega_{\xi}\left(\delta_{n-1}\right)}{C_{2} \delta_{n} \varphi^{*-1}\left(h\left(C_{2} \delta_{n-1}\right)\right)}>u\right)=\mathrm{P}\left(\frac{\omega_{\xi}\left(\delta_{n-1}\right)}{C_{2} \delta_{n-1}}>u \frac{\delta_{n}}{\delta_{n-1}} \varphi^{*-1}\left(h\left(C_{2} \delta_{n-1}\right)\right)\right)$.

Обозначим

$$
u_{n}=u \frac{\delta_{n}}{\delta_{n-1}} \varphi^{*-1}\left(h\left(C_{2} \delta_{n-1}\right)\right) .
$$

По условию г) найдутся такие $n_{0}$ и последовательность $y_{n}$, что выбирая $\delta_{n}=y_{n} / C_{2}$, при всех $n \geqslant n_{0}$ будем иметь

$$
\delta_{n} \geqslant \frac{1+\varepsilon / 2}{1+\varepsilon} \delta_{n-1} .
$$

Таким образом,

$$
u_{n} \geqslant u \frac{1+\varepsilon / 2}{1+\varepsilon} \varphi^{*-1}\left(h\left(C_{2} \delta_{n-1}\right)\right) .
$$

Очевидно, что $u_{n} \rightarrow \infty$ при $n \rightarrow \infty$. Теперь

$$
P_{n}(u) \leqslant C_{1} N\left(T, \frac{d}{C_{2} \delta_{n-1}}, \pi\left(u_{n}\right)\right) \exp \left(-\varphi^{*}\left(u_{n}\right)\right) .
$$

В силу локальной однородности СП $\xi(t)$ имеем

$$
\begin{aligned}
N\left(T, \frac{d}{C_{2} \delta_{n-1}}, \pi\left(u_{n}\right)\right) & =\exp \left(H\left(T, \frac{d}{C_{2} \delta_{n-1}}, \pi\left(u_{n}\right)\right)\right) \\
& \leqslant C_{5} \exp \left(h\left(C_{2} \delta_{n-1}\right)+h\left(\pi\left(u_{n}\right)\right)\right)
\end{aligned}
$$

где $C_{5}=N(T, d, 1)$. Согласно условию в) теоремы

$$
h\left(\pi\left(u_{n}\right)\right) \leqslant \frac{\varepsilon / 4}{1+\varepsilon / 2} \varphi^{*}\left(u_{n}\right)
$$

при достаточно больших $n$. Поэтому

$$
P_{n}(u) \leqslant C_{6} \exp \left(h\left(C_{2} \delta_{n-1}\right)-\frac{\varepsilon / 4}{1+\varepsilon / 2} \varphi^{*}\left(u_{n}\right)\right),
$$

где $C_{6}=C_{1} C_{5}$.

Докажем сначала (5). Для этого оценим $P_{n}(1+\varepsilon)$, используя лемму $1: P_{n}(1+\varepsilon) \leqslant$ $C_{6} \exp \left(-(\varepsilon / 4) h\left(C_{2} \delta_{n-1}\right)\right)$. В силу выбора $\delta_{n}$ и согласно условию г) теоремы

$$
\sum_{n} P_{n}(1+\varepsilon) \leqslant C_{6} \sum_{n} \exp \left(-\frac{\varepsilon}{4} h\left(y_{n}\right)\right)<\infty,
$$


так что (5) следует из леммы Бореля-Кантелли. Теперь докажем (4). Очевидно, что $P_{T}(u) \leqslant \sum_{n} P_{n}(u)$. Поэтому при всех $\varepsilon \leqslant \varepsilon_{0}$, обозначая

$$
C_{7}=\frac{1+\varepsilon_{0} / 4}{1+\varepsilon_{0}}
$$

получим

$$
\begin{aligned}
P_{T}(u) & \leqslant \sum_{n=1}^{\infty} \exp \left(-\left(C_{7} u-1\right) h\left(y_{n-1}\right)\right) \\
& =\exp \left(-\left(C_{7} u-1\right) h\left(y_{1}\right)\right)\left(1+\sum_{n=1}^{\infty} \exp \left(-\left(C_{7} u-1\right) h\left(y_{n-1}\right)-h\left(y_{1}\right)\right)\right) .
\end{aligned}
$$

Последний ряд по условию г) сходится при любом $u \geqslant u_{0}>1 / C_{7}$. Обозначив произведение величины, стоящей в фигурных скобках, на $\exp \left(h\left(y_{1}\right)\right)$ через $C_{3}$, a $C_{7} h\left(y_{1}\right)-$ через $C_{4}$, получим (4). Теорема 1 полностью доказана.

Рассмотрим теперь нецентрированное поле $\tau(t)=\xi(t)+a(t)$, где $a(t)$ - некоторая неслучайная функция.

ТЕОРема 2. Пусть выполнены условия теоремы 1 и, кроме того, существует некоторая константа $C_{8}$ такая, что для любых $t, s \in T$ выполнено соотношение

$$
|a(t)-a(s)| \leqslant C_{8} d(t, s) .
$$

Тогда с вероятностью 1

$$
\varlimsup_{\delta \rightarrow 0} \frac{\omega_{\tau}(\delta)}{g(\delta)} \leqslant 1
$$

и при этом

$$
P_{T}(u)=\mathrm{P}\left(\sup _{\delta \in\left(0, \delta_{0}\right)} \frac{\omega_{\tau}(\delta)}{g(\delta)}>u\right) \leqslant C_{3} e^{-C_{9} u} .
$$

ДокАЗАТЕЛЬСТво. По условию теоремы $\omega_{a}(\delta) \leqslant C_{8} d(t, s)$. Как следует из теоремы $1, \omega_{\xi}(\delta) \leqslant \eta C_{2} \delta \varphi^{*-1}\left(h\left(C_{2} \delta\right)\right)$, где

$$
\eta=\sup _{\delta \in\left(0, \delta_{0}\right)} \frac{\omega_{\xi}(\delta)}{C_{2} \delta \varphi^{*-1}\left(h\left(C_{2} \delta\right)\right)}
$$

- случайная величина с экспоненциально убьвающим "хвостом" распределения. Поэтому

$$
\omega_{\tau}(\delta) \leqslant \omega_{\xi}(\delta)+\omega_{a}(\delta) \leqslant C_{2} \delta\left(\eta \varphi^{*-1}\left(h\left(C_{2} \delta\right)\right)+C_{8}\right) \leqslant \eta C_{2} \delta \varphi^{*-1}\left(h\left(C_{2} \delta\right)\right)(1+\beta(\delta)),
$$

где $\beta(\delta) \rightarrow 0$ при $\delta \rightarrow 0$.

Согласно (4) $P_{T}(u) \leqslant C_{3} \exp \left(-C_{4} u /\left(1+\beta\left(\delta_{0}\right)\right)\right)$. Обозначая теперь через $C_{9}$ величину $C_{4} u /\left(1+\beta\left(\delta_{0}\right)\right)$, приходим к $(8)$. Соотношение $(7)$ сразу же вытекает из теоремы 1. Итак, теорема 2 доказана. 


\section{3. Примеры.}

ПримеР 1. Пусть $h(\varepsilon) \leqslant h_{0}+\varkappa \ln (1 / \varepsilon)$, тогда если $\ln \varphi^{* \prime}(u)=o\left(\varphi^{*}(u)\right)$ при $u \rightarrow \infty$ и вьполнено условие $(6)$, то

$$
\mathrm{P}\left(\varlimsup_{\delta \rightarrow 0} \frac{\omega_{\tau}(\delta)}{\delta \varphi^{*-1}(\varkappa \ln (1 / \delta))} \leqslant C_{2}\right)=1
$$

и при этом

$$
\mathrm{P}\left(\sup _{\delta \in\left(0, \delta_{0}\right)} \frac{\omega_{\tau}(\delta)}{C_{2} \delta \varphi^{*-1}\left(\varkappa \ln \left(1 /\left(C_{2} \delta\right)\right)\right)}>u\right) \leqslant C_{3} e^{-C_{9} u}
$$

ПримеР 2. Пусть $h(\varepsilon) \leqslant C_{10} \varepsilon^{-\beta}, \beta \in(0,1)$ и при $u \rightarrow \infty u^{\beta} \varphi^{* \prime \beta}(u)=o\left(\varphi^{*}(u)\right)$. Тогда если вьполнено условие (6), то для $g(\delta)=C_{2} \delta \varphi^{*-1}\left(C_{10}\left(C_{2} \delta\right)^{-\beta}\right)$ справедливы формулы (4) и (5).

4. Нижняя оценка. Покажем на примере, что логарифмический множитель в $g(\delta)$ существенен, т.е. что $g(\delta)$ дает точную по порядку асимптотику (в смысле верхнего предела) функции $\omega_{\tau}(\delta)$.

Пусть $\xi(t)$ - центрированньй гауссовский стационарньй процесс с ковариационной функцией $r(t)=e^{-|t|^{\alpha}}, \alpha \in(0,2], t \in[0,1]$. Рассмотрим классический сплайн первого порядка: $t_{i}$ - минимальная $\delta$-сеть отрезка $[0,1]$ в метрике $d, i=1, \ldots, n$. Тогда

$$
\widehat{\xi}(t)=\xi\left(t_{i}\right)+\frac{t-t_{i}}{t_{i+1}-t_{i}} \xi\left(t_{i+1}\right) \quad t \in\left[t_{i}, t_{i+1}\right] .
$$

При этом $d(t, s)=\sqrt{\mathrm{D}(\xi(t)-\xi(s))} \sim \sqrt{2}|t-s|^{\alpha / 2}, t-s \rightarrow 0, h(\varepsilon) \leqslant h_{0}+(2 / \alpha) \ln (1 / \varepsilon)$, $n \leqslant \varepsilon^{-2 / \alpha}$.

Обозначим

$$
\eta_{\delta}(k)=\left(\xi\left(\frac{k+0.5}{n}\right)-0.5\left(\xi\left(\frac{k}{n}\right)+\xi\left(\frac{k+1}{n}\right)\right)\right) \frac{n^{\alpha / 2}}{\sqrt{2}} .
$$

Прямым вычислением легко показать, что коэффициент корреляции

$$
\rho(k)=\rho\left(\eta_{\delta}(0), \eta_{\delta}(k)\right) \leqslant \frac{C_{11}}{k^{\alpha / 2}}, \quad \mathrm{D} \eta_{\delta}(k) \sim 1, \quad \delta \rightarrow 0 .
$$

Очевидно, что

$$
\varlimsup_{\delta \rightarrow 0} \frac{\omega_{\xi}(\delta)}{\delta \sqrt{(4 / \alpha) \ln (1 / \delta)}} \geqslant \varlimsup_{n \rightarrow \infty} \frac{\max _{1 \leqslant k \leqslant n} \eta_{\delta}(k)}{\sqrt{2 \ln n}} .
$$

Согласно [11], последний предел с вероятностью 1 равен 1 . В то же время, из (7) вытекает неравенство

$$
\left.\varlimsup_{\delta \rightarrow 0} \frac{\omega_{\xi}(\delta)}{\delta \sqrt{(4 / \alpha) \ln (1 / \delta)}} \leqslant 1 \quad \text { (с вероятностью } 1\right) .
$$

Таким образом, построен пример, в котором

$$
\mathrm{P}\left(\varlimsup_{\delta \rightarrow 0} \frac{\omega_{\xi}(\delta)}{g(\delta)}=1\right)=1 .
$$


Покажем на том же примере, что оценка $(8)$ вероятности $P_{T}(u)$ не улучшаема по порядку показателя экспоненты:

$$
\begin{aligned}
P_{T}(u) & \geqslant \mathrm{P}\left(\sup _{n \geqslant n_{0}} \frac{\max _{1 \leqslant k \leqslant n} \eta_{\delta}(k)}{\sqrt{2 \ln n}}>u\right) \\
& \geqslant \sup _{n \geqslant n_{0}} \mathrm{P}\left(\frac{\max _{1 \leqslant k \leqslant n} \eta_{\delta}(k)}{\sqrt{2 \ln n}}>u\right) \geqslant \lim _{n \rightarrow \infty} \mathrm{P}\left(\frac{\max _{1 \leqslant k \leqslant n} \eta_{\delta}(k)}{\sqrt{2 \ln n}}>u\right) .
\end{aligned}
$$

Согласно [12, с. 100], последний предел равен $1-\exp (-\exp (-u))$, что при $u \rightarrow \infty$ эквивалентно $\exp (-u)$.

\section{СПИСОК ЦИТИРОВАННОЙ ЛИТЕРАТУРЫ}

[1] Беляев Ю. К., Симонян А. Х., Красавкина В. А. Квантование по времени реализаций недифференцируемых гауссовских процессов // Изв. АН СССР. Сер. техн. киберн. 1976. № 4. C. $139-147$.

[2] Фролов А. С., Ченцов Н. Н. О вычислении методом Монте-Карло определенных интегралов, зависящих от параметра // ЖВМиМФ. 1962. Т. 2. № 4. С. 714-717.

[3] Дмитровский В. А., Островский Е. И. Оценка погрешности метода зависимых испытаний // ЖВМиМФ. 1976. Т. 16. № 5. С. 1312-1316.

[4] Островский Е.И. Обобщение нормы Булдьпина-Козаченко и центральная предельная теорема в банаховом пространстве // Теор. вероятн. и ее примен. 1982. Т. 27. № 3. С. 618 .

[5] Козаченко Ю. В., Островский Е. И. Банаховы пространства случайных величин типа субгауссовских // Теор. вероятн. и матем. статистика. № 32. Киев: КГУ, 1985. С. 42-53.

[6] Рокафеллар В. Выпуклый анализ. М.: Мир, 1969.

[7] Островский Е. И. Двусторонние экспоненциальные оценки для распределения максимума случайных полей // УМН. 1992. Т. 47. № 5. С. 225-226.

[8] Ostrovskii E. I. Two-sides exponential inequalitiy for the distribution of the norm of Banach space valued random variables // New Trends in Probability and Statistics. V. 1. Utrecht-Tokyo: Mokslas, 1993. P. 246-254.

[9] Островский Е.И. Регрессионные сплайны // Исследование нелинейных моделей матем. физики. Обнинск: ИАТЭ, 1990. С. 103-110.

[10] Стечкин С. Б., Субботин Ю.Н. Сплайны в вычислительной математике. М.: Наука, 1976.

[11] Berman S. M. Limit theorem for the maximum term in stationary sequences // Ann. Math. Stat. 1964. V. 35. P. 502-516.

[12] Лидбеттер М., Ротсен Х., Линдгрен Т. Экстремумы случайных последовательностей и процессов. М.: Мир, 1989. 